\title{
UNIFORM DISTRIBUTION OF HASSE INVARIANTS
}

\author{
R.A. MOLLIN \\ Department of Mathematics and Statistics \\ The University of Calgary \\ Calgary, Alberta \\ T2N 1 N4 Canada \\ (Received June 4, 1984)
}

\begin{abstract}
I. Schur's study of simple algebras around the turn of the century, and subsequent investigations by R. Brauer, E. Witt and others, were later reformulated in terms of what is now called the Schur subgroup of the Brauer group. During the last twenty years this group has generated substantial interest and numerous palatable results have ensued. Among these is the discovery that elements of the Schur group satisfy uniform distribution of Hasse invariants. It is the purpose of this paper to continue an investigation of the latter concept and to highlight certain applications of these results, not only to the Schur group, but also to embeddings of simple algebras and extensions of automorphisms, among others.
\end{abstract}

KEY WORDS AND PHRASES. Hasse invariant, Schur index, uniform distribution, embedding, simple algebra.

1980 A.M.S. Subject Classification: Primary 16A26, $16 A 48$ Secondary $12 B 99$.

\section{INTRODUCTION:}

For a field $\mathrm{K}$ of characteristic zero, the Schur subgroup $\mathrm{S}(\mathrm{K})$ of the Brauer group $\mathrm{B}(\mathrm{K})$ consists of all equivalence classes [A] which contain a $\mathrm{K}$-isomorphic copy of a simple summand of the group algebra KG for some finite group $G$. We restrict our attention to fields of characteristic zero since $S(K)$ is trivial when $K$ has non-zero characteristic. This follows from Weddeburn's well-known theorem which states that a finite-dimensional division algebra over a finite field is itself a field.

It was shown in Benard et al [1, Theorem 1, p.380] that when $K / Q$ is finite abelian and $[A] \in S(K)$ with index $m$ then: (1.1) $\epsilon_{m}$, a primitive $m^{\text {th }}$ root of unity, is in $K$, and (1.2) suppose $\sigma \in G(K / Q)$, the Galois group of $K / Q$, with $\epsilon_{m}^{\sigma}=\epsilon_{m}^{b}$. If $P$ is $K-p r i m e$ then the following relationship holds between the Hasse invariants of $A$ : $\operatorname{inv}_{P}(A) \equiv b_{S} \operatorname{inv}_{P} \sigma(A)(\bmod 1)$.

Algebras satisfying (1.1)-(1.2) are called algebras with uniformly distributed Hasse invariants. Such algebras can be shown to form a subgroup $U(K)$ of $B(K)$, and therefore we have $S(K)$ as a subgroup of $U(K)$. For $K / Q$ abelian we have studied the relationship between $S(K)$ and $U(K)$ in Mollin $[2,9]$. However the study of $U(K)$ has proved valuable from several vantage points not directly related to the group itself. In particular certain tools have been developed in the aforementioned papers which have proved to be valuable in answering related open questions. Some examples are as follows. In 
Mollin [10] we proved a conjecture which stated that if the center of a finite dimensional division algebra, D, contains no nontrivial odd order roots of unity then all finite odd order subgroups of the multiplicative group of D are cyclic. This problem is related to a conjecture of I.N. Heistein and results of S. Amitsur. Moreover in Mollin [11] we provided sufficient conditions for the existence of a splitting field $L$ of an absolutely irreducible character $x$ of a finite group of exponent $n$, such that $L=Q\left(\epsilon_{n}\right)$ and $|L: Q(X)|=m_{Q}(X)$, the Schur index of $X$ over $Q$. This result is a natural outcropping of $R$. Brauer's well-known theorem stating that $Q\left(\epsilon_{n}\right)$ splits $\chi$. Furthermore, in Mollin [12 - 14] we used the aforementioned techniques to answer specific questions pertaining to the structures of division algebras, the Schur index, and class field theory proper.

Given the above, it is natural to seek a more general context for uniform distribution. In Mollin [15] we observed that $U_{F}(K)$, for $K / F$ a finite Galois extension of number fields, could be defined as those elements of $B(K)$ satisfying (1.1) and (1.2), where $Q$ is replaced by $F$. Moreover, as with the abelian case, we have that $U_{F}(K)$ is a subgroup of $\mathrm{B}(\mathrm{K})$ and $\mathrm{S}(\mathrm{K})$ is again a subgroup of $\mathrm{U}_{\mathrm{F}}(\mathrm{K})$. Furthermore another property which was proved in Bernard [16] to hold for $S(K)$ carries over to $U_{F}(K)$. We isolate this property since it will be of independent interest later: (1.3) Let $\mathrm{K} / \mathrm{F}$ be normal and suppose $[A] \in U_{F}(K)$. If $\hat{P}$ and $\hat{Q}$ are $K$-primes above an $F$-prime $P$ then $A \otimes_{K} K_{P}$ and $A \hat{K}_{K} K_{\hat{Q}}$ have the same index, where $K_{\hat{p}}$ (respectively $K_{\hat{Q}}$ ) denotes the completion of $\mathrm{K}$ at $\hat{P}$ (respectively $\hat{Q}$ ). The common value of indices $A \otimes_{K} K_{\hat{p}}$ for all K-primes $\hat{P}$ above $P$ is called the $P$-local index of $A$, denoted ind $p(A)$.

Now we ask whether $U_{F}(K)$ may somehow be salvaged when $K / F$ is any (not necessarily normal) extension of number fields. We define $U_{F}(K)$ to consist of those $[A] \in B(K)$ such that $\left[A \otimes_{K} L\right] \in U_{F}(L)$, where $L$ is the normal closure of $K$ over $F$. We call $U_{F}(K)$ the group of algebras with uniformly distributed invariants over $\mathrm{K}$ relative to $\mathrm{F}$. It is straightforward to check that $U_{F}(K)$ is a subgroup of $B(K)$ and that $S(K)$ is in turn a subgroup of $U_{F}(K)$. The latter fact follows from the fact that $S(K) \infty_{K} L$ is in $U_{F}(L)$. However when $K \neq L, U_{F}(K)$ differs markedly from $U_{F}(L)$. In particular the following example shows that (1.3) fails to hold for $U_{F}(K)$.

EXAMPLE 1.4. Let $K=Q\left(\theta, \epsilon_{4}\right)$ where $\theta$ is a real root of $f(x)=x^{3}-2$ and let $F=Q$. Then the normal closure of $K$ over $F$ is $L=Q\left(\theta, \epsilon_{12}\right)$. It can be verified that the prime 29 splits into four unramified $K$-primes $P_{1}, P_{2}, P_{3}$ and $P_{4}$ with $P_{i}$ for $i=1,2$ having inertial degrees equal to one over $Q$, whereas $P_{i}$ for $i=3,4$ have inertial degrees equal to two over $Q$.

We now define a central simple $\mathrm{K}$-algebra, $\mathrm{A}$, as follows: let $\operatorname{inv}_{P_{i}}(A)=1 / 2$ for $i=3,4 ; \operatorname{inv}_{P_{1}}(A)=1 / 4$ and $\operatorname{inv}_{P_{2}}(A)=3 / 4$ while inv $_{Q}(A)=0$ for all $\mathrm{K}$-primes $2 \neq P_{i}$ where $i=1,2,3,4$. By the Hasse sum theorem (see Reiner [17]) we are guaranteed that $[A] \in B(K)$. Now let $\hat{P}_{i}$ be an $L$-prime above $P_{i}$ for $i=1,2,3,4$. It can be verified that $\hat{P}_{i}$ for $i=1,2$ have inertial degrees equal to 2 in $L$ over $K$ and $\hat{P}_{i}$ for $i=3,4$ have inertial degrees equal to 1 in $L$ over $K$. Hence we obtain that $\operatorname{inv}_{\hat{p}}\left(A \otimes_{K} L\right)$ $=1 / 2$ for $i=1,2,3,4$. Therefore by $(1.1)-(1.2)$ we have that $\left[A \Theta_{K} L\right] \in U_{Q}(L)$, and so [A] $\in U_{Q}(K)$. However the index of $A \phi_{K} K_{P_{2}}$ is four, whereas the index of $A \otimes_{K} K_{P_{3}}$ 
is two, contradicting (1.3). This completes the example.

The first main result of this paper is to provide a generalization of a theorem of E. Witt using $U_{F}(K)$ as a tool. From this result we will see that (1.1)-(1.2) fail to hold for $U_{F}(K)$ when $K \neq L$. Furthermore we use this generalized Witt theorem to develop further properties of $J_{F}(K)$. Moreover we obtain necessary and sufficient conditions, in terms of the arithmetic of the fields under consideration, for an element to exist in $U_{F}(K)$ with a given index. Several related properties are also developed.

Finally we generalize the concept of K-adequacy introduced in Fein et al [18] and link it to $U_{F}(K)$ via the arithmetic of the underlying fields in a sequence of results. 2. BASIC REFERENCES

For basic properties of number fields used in this paper we refer the reader to Marcus [19]. For fundamental results concerning the Schur subgroup of the Brauer group the reader should consult Yamada [20]. For information pertaining to properties of algebras used herein, and especially the classification of the Brauer group of a number field via Hasse invariants see the beautifully written Reiner [17]. Any concepts not described in greater detail in this paper may be found in earlier work Mollin [2 - 15].

\section{UNIFORM DISTRIBUTION}

THECREM 3.1. Suppose $[A] \in U_{F}(K)$ where $K / F$ is an extension of number fields. Let $P$ be a K-prime with $\operatorname{inv}_{P}(A)>0$, and let $\hat{P}$ be an L-prime above $P$ where $L$ is the normal closure of $K / F$. Set $g=$ g.c.d. $\left(m,\left|L_{\hat{p}}: K_{P}\right|\right)$, where $m$ is the index of $A \dot{K}_{K} K_{P}$ Then $\epsilon_{\mathrm{m} / \mathrm{g}}$ is in $\mathrm{K}$ and $P \cap F$ is completely split in $F\left(\epsilon_{\mathrm{m} / \mathrm{g}}\right)$.

PROOF. Let $\sigma \in G(L / K)$ and let $\hat{P}$ be an L-prime with $\hat{P} \cap \mathrm{K}=P$. Therefore $\operatorname{inv}_{\hat{p}}\left(A \otimes_{K} L\right)=b_{\sigma} \operatorname{inv} \hat{p}^{\sigma}\left(A \otimes_{K} L\right)$ where $\epsilon_{m}^{\sigma}=\epsilon_{m}^{b}$. Hence: $\left|L_{\hat{p}}: K_{p}\right| \operatorname{inv}_{p}(A) \equiv b_{\sigma}\left|L_{\hat{p}}{ }^{\sigma}: K_{p}\right| \operatorname{inv}_{P}(A)(\bmod 1)$. Thus $b_{\sigma} \equiv 1(\bmod \mathrm{m} / g)$, and so $\sigma$ fixes $\epsilon_{\mathrm{m} / \mathrm{g}}$ for all $\sigma \in \mathrm{G}(\mathrm{L} / \mathrm{K})$; i.e. $\epsilon_{\mathrm{m} / \mathrm{g}}$ is in $\mathrm{K}$.

Since we have: $\operatorname{inv}_{\hat{p}}\left(A \otimes_{K} L\right) \equiv\left|L_{\hat{p}}: K_{p}\right| \operatorname{inv}_{p}(A)(\bmod 1)$ then $\operatorname{ind}_{p}\left(A \otimes_{K} L\right)=\mathrm{m} / \mathrm{g}$. Hence by Mollin [15, Theorem 2.3, p.251], $P \cap F$ is completely split in $F\left(\epsilon_{m / g}\right)$.

For completeness sake we state Witt's results [21, Satz 10, Satz 11, p.243] in succinct form as a corollary which is immediate from the theorem.

COROLLARY 3.2. If $K / Q$ is finite abelian, $[A] \in S(K)$ and $P$ is an odd prime with ind $_{p}(A)=m$ then $p \equiv 1(\bmod m)$. If $p=2$ then ind $(A)=1$ or 2 .

We maintain the notation of the Theorem 3.1 in the following results. The first result, which is immediate, generalizes Mollin [15, Corollary 2.4, p.254].

COROLLARY 3.3 Let $\mathrm{m} / \mathrm{g}=\mathrm{p}^{\mathrm{a}}$ where $\mathrm{p}$ is a prime and suppose $\epsilon_{\mathrm{p}} \mathrm{n}$ is the largest $p$-power root of unity in $K$. If $P$ is completely split in $F\left(\epsilon_{p} c\right)$ but not in $F\left(\epsilon_{p} c+l\right)$ then $\mathrm{a} \leq \min \{\mathrm{n}, \mathrm{c}\}$.

The next result which will prove to be useful later in the paper generalizes Mollin [15, Corollary 2.5, p.254].

COROLlARY 3.4. Suppose $P$ and $Q$ are $K$-primes with $P \cap F=Q \cap F$ and $\left|L_{\hat{p}}: K_{P}\right|=$ $\left|\mathrm{L}_{\hat{Q}}: \mathrm{K}_{\Omega}\right|$ where $\hat{P}$ (respectively $\hat{Q}$ ) is any L-prime above $P$ (respectively $\left.Q\right)$. If $[A] \epsilon U_{F}(K)$ then $\operatorname{inv}_{P}(A)=\operatorname{inv}_{Q}(A)$ if and only if $P \cap F\left(\epsilon_{m / g}\right)=Q \cap F\left(\epsilon_{m / g}\right)$. 
PROOF. Suppose $\sigma \in G(L / F)$ such that $\hat{p}^{\hat{\sigma}}=\hat{Q}$. We have $\operatorname{inv}_{\hat{p}}\left(A \dot{\alpha}_{K} L\right) \equiv b_{\sigma}$ $\operatorname{inv}_{\hat{p}}^{\sigma}\left(A \approx_{K}\right.$ L) $(\bmod 1)$ where $\epsilon_{\mathrm{m} / g}^{\sigma}=\epsilon_{\mathrm{m} / g}^{b_{\sigma}}$. Thus we have by Mollin $[4,(2.3), p .276]$ that: $\left|\mathrm{L}_{\hat{p}}: \mathrm{K}_{p}\right| \operatorname{inv}_{p}(A) \equiv \mathrm{b}_{\sigma}\left|\mathrm{L}_{\hat{Q}}: \mathrm{K}_{2}\right| \operatorname{inv}_{2}(\mathrm{~A})(\bmod 1)$.

Since $\left|\mathrm{L}_{\hat{p}}: \mathrm{K}_{p}\right|=\left|\mathrm{L}_{\hat{Q}}: \mathrm{K}_{2}\right|$ then we have that inv ${ }_{p}(\mathrm{~A})=\operatorname{inv}_{2}(\mathrm{~A})$ if and only if $b_{\sigma} \equiv 1$ $(\bmod \mathrm{m} / \mathrm{g})$ which in turn holds if and only if $\sigma \in \mathrm{G}\left(\mathrm{L} / \mathrm{F}\left(\epsilon_{\mathrm{m} / \mathrm{g}}\right)\right)$. However by theorem 3.1, $P \cap F$ is completely split in $F\left(\epsilon_{\mathrm{m} / g}\right)$. Hence $\sigma \epsilon G\left(\mathrm{~L} / \mathrm{F}\left(\epsilon_{\mathrm{m} / \mathrm{g}}\right)\right)$ if and only if $P \cap F\left(\epsilon_{m / g}\right)=P^{\sigma} \cap F\left(\epsilon_{/ g}\right)$ Q.E.D.

We note that Theorem 3.1 shows that (1.1) does not hold ior $U_{F}(K)$ when $K / F$ is nonnormal. Moreover (1.2) does not generalize to $U_{F}(K)$ where of $(1.1)$ is interpreted as an embedding of $K$ into the complex field $C$. This may be illustrated by considering example $(1 . \dot{4})$ with $\sigma \in C(L / K)$ and $\hat{P}_{y}^{v}=\hat{P}_{4}$. However inv $P_{2}(A)=3 / 4$ inv $_{P_{3}}(A)$ $\equiv 1 / 2(\bmod 1)$.

Now, Theorem 3.1 is the "best possible" generalization of Witt's results in the sense that we cannot hope for $P \cap F$ to be completely split in $F\left(\epsilon_{n}\right)$ for $m \geq n>m / g$ in general. The following example depicts this fact.

EXAMPLE 3.5. Let $\mathrm{a}$ be a real root of $\mathrm{f}(\mathrm{x})=\mathrm{x}^{1 \epsilon}-2$, and let $F=Q(\theta)$. If $K=Q\left(,, \epsilon_{8}\right)$ then $L=Q\left(, \epsilon_{16}\right)$ is the normal closure of $K$ over $F$. Let $P$ be an F-prime above 5. Then it can be verified that $P$ splits into two unramified K-primes $\hat{P}_{1}$ and $\hat{P}_{2}$ each having inertial degree two over $P$. Define: inv $\hat{P}_{1}(A)=1 / 8$, and inv $\hat{P}_{2}(A)=$ $-1 / 8$ while $\operatorname{inv}_{\hat{O}}(\mathrm{~A})=0$ for all k-primes $\hat{\imath} \neq \hat{P}_{1}, \hat{P}_{2}$. Then by the Hasse sum $\mathrm{T}$ heorem $[A] \in B(K)$. Now since there exists exactly one $L$-prime $\hat{Q}_{i}$ above $\hat{P}_{i}$ for $i=1,2$, each with inertial degree equal to 2 then $\operatorname{inv}_{\hat{Q}_{1}}\left(A Q_{K} L\right)=1 / 4$ and $\operatorname{inv}_{\hat{n}_{2}}\left(A Q_{K} L\right)=-1 / 4$. Thus by construction $[A] \in U_{F}(K)$. However, although the index of $A \otimes_{K} K_{\hat{p}}$ is $8, P$ is not completely split in $F\left(\epsilon_{8}\right)=K$. This completes the example.

It is natural to ask whether the converse of Theorem 3.1 holds since we would then have a criterion, in terms of the arithmetic of $\mathrm{K}$ and $\mathrm{F}$, for the existence of an element in $U_{F}(K)$. Unfortunately the converse fails to hold as the following counterexample illustrates.

EXAMPLE 3.6. Let $K=Q\left(\theta_{1}\right), F=Q$, and $L=Q\left(\theta_{2}, \epsilon_{3}\right)$ where $\theta_{1}$ is a real root of $f(x)=x^{3}-2$ and $\theta_{2}$ is a real root of $g(x)=x^{3}-11$. Then 2 splits into two $K$-primes $P_{1}$ and $P_{2}$ with inertial degrees one and two respectively over $F$. Hence $P_{1}$ splits into two L-primes $\hat{P}_{1}$ and $\hat{P}_{2}$ each with inertial degree 2 over $K$; and $P_{2}$ has one L-prime $\hat{P}_{3}$ above it with inertial degree one over $K$. Now, if there exists $[A] \in U_{F}(K)$ with $\operatorname{inv}_{P_{2}}(A)=1 / 2$ then $\left[A \otimes_{K} L\right] \in U_{F}(L)$. Therefore: $i n \hat{P}_{3}\left(A Q_{K} L\right) \equiv\left|L_{P_{3}}: K_{P_{2}}\right| i n P_{P_{2}}(A)$ $\equiv \operatorname{inv}_{P_{2}}(A) \equiv 1 / 2(\bmod 1)$. Therefore $\operatorname{ind}_{2}\left(A \otimes_{K} L\right)=2$ is forced. Thus: $\operatorname{inv}_{\hat{P}_{i}}\left(A \otimes_{K} L\right)=1 / 2$ for $i=1,2,3$. However by Mol1in [15, Lemma 2.8, p. 259] we must have $\sum_{i=1}^{3} i n \hat{p}_{i}\left(A \otimes_{K} L\right) \equiv 0(\bmod 1)$, a contradiction which completes the example.

Now we demonstrate that under a suitable restriction we do get sufficient conditions in terms of the arithmetic of $F, K$ and $L$ to guarantee the existence of an element in $U_{F}(K)$.

THEOREM 3.7. Let $\mathrm{L}$ be the normal closure of $\mathrm{K} / \mathrm{F}$, an extension of number fields. Suppose that $\epsilon_{n}$ is the largest root of unity in $F$ with $n$ 
(1) $\epsilon_{m}$ is in $K$ where $n$ divides $m$ and;

(2) $\hat{P}^{m}$ is a K-prime such that $\hat{P} \cap F=P$ is completely split in $F\left(\epsilon_{m}\right)$, and

(3) g.c.d. ( $r,|L: K|)=1, \quad$ where $r=\left\{\begin{array}{ll}m / n & \text { if } m>n \\ m & \text { if } m=n\end{array}\right\}$.

then there exists $[A] \in U_{F}(K)$ with the index of $A \otimes_{K} K_{p}^{\hat{p}}$ equal to $r$.

PROOF. By [15, Theorem 2.7, p.256] we have the existence of an element [B] $\epsilon$ ${ }_{F}(L)$ with ind $P(B)=r$. Now, let $I(B)=\{F$-primes $P$ : ind $(B)>1\}$. Suppose that

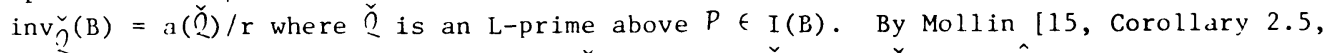
P. 254] we have that, for any L-prime $\check{R}$ such that $\check{n} \cap \mathrm{K}=\check{\mathrm{R}} \cap \mathrm{K}=\hat{n}$ then, inv $\check{R}(\mathrm{~B})=$

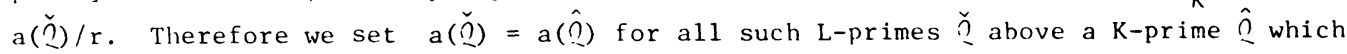
lies above a given $P \in I(B)$. Now we define a K-central simple algebra A as follows. For each $P \in I(B)$, let $\operatorname{inv}_{\hat{O}}(\mathrm{~A})=\mathrm{a}(\hat{\Omega}) P(\hat{\cap}) / \mathrm{r}$, for a $11 \mathrm{~K}$-primes $\hat{Q}$ above $P$, and 1 et $\operatorname{inv}_{\hat{S}}(A)=0$ for all $\mathrm{K}$-primes $\hat{S}$ not above primes in $\mathrm{I}(\mathrm{B})$, where $2(\hat{\Omega})=|\mathrm{L}: \mathrm{K}| /\left|\mathrm{L}_{\hat{\Omega}}: \mathrm{K}_{\hat{O}}\right| \cdot$ By (3) of the hypothesis we have that the index of $A \otimes_{K} K_{n}$ is equal to $r$. Moreover:

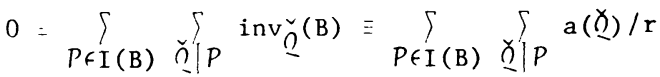

$$
\begin{aligned}
& \equiv \sum_{P \in I(B)} \hat{O} \mid P a(\hat{Q}) \ell(\hat{n}) / r \equiv \sum_{P \in I(B)} \sum_{\hat{O} \mid P} i n v \hat{n}(A)(\bmod 1) .
\end{aligned}
$$

Therefore $[A] \in B(K)$. Now, if $\sigma \in G(L / F)$ then:

$$
\begin{aligned}
& \operatorname{inv}_{\bigcap}\left(A \otimes_{K} L\right) \equiv|L: K| a(\hat{)}) / r \equiv|L: K| i n v \check{Q}(B) \\
& \equiv|\mathrm{L}: \mathrm{K}| \mathrm{b}_{\sigma} \operatorname{inv} \hat{\Omega}^{\sigma}(\mathrm{B}) \equiv|\mathrm{L}: \mathrm{K}| \mathrm{b}_{\sigma} \mathrm{a}\left(\Omega^{\sigma}\right) / \mathrm{r} \equiv \mathrm{b}_{\sigma} \operatorname{inv}_{\Omega^{(\sigma}}\left(\mathrm{A} \mathrm{Q}_{\mathrm{K}} \mathrm{L}\right)(\bmod 1)
\end{aligned}
$$

where $\hat{Q}^{\sigma}$ is a $K$-prime below $\hat{Q}^{\sigma}$. Hence $[A] \in U_{F}(K)$. Q.E.D.

We note that Theorem 3.7 generalizes Mol1in [15, Theorem 2.7, p.256].

The final result of this section is an interesting result which generalizes Mollin [15, Lemma 3.1, P.262] and simplifies the proof thereof. Moreover we note that it is possible to use the following result to generalize Mollin [15, Theorem 2.10, P. 260].

THEOREM 3.8. Let $K_{i} / F$ for $i=1,2$ be extensions of number fields with $N_{1}$ being the normal closure of $K_{1} / F$ and $N_{2}$ being the normal closure of $K_{1} K_{2} / F$. If [A] $\epsilon U_{F}\left(K_{1}\right)$ with index $m$ and g.c.d. $\left(m,\left|N_{1} N_{2}: N_{2}\right|\right)=1$ then $\left[A Q_{K_{1}} K_{1} K_{2}\right] \in U_{F}\left(K_{1} K_{2}\right)$.

PROOF. Let $g=g \cdot c \cdot d \cdot\left(m,\left|N_{1} N_{2}: K_{1}\right|\right)$, and let $\sigma \in G\left(N_{2} / F\right)$ with $\epsilon_{m / g}=\epsilon_{m / g}^{b}$. Denote an extension of $\sigma$ to $G\left(N_{1} N_{2} / F\right)$ by $\hat{\sigma}$. Since $\left[A \otimes_{K_{1}} N_{1}\right] \in U_{F}\left(N_{l}\right)$ implies that $\left[A \otimes_{K_{1}} N_{1} N_{2}\right] \in U_{F}\left(N_{1} N_{2}\right)$ then we have inv $\left.\hat{p}^{\left(A \otimes_{K_{1}}\right.} N_{1} N_{2}\right) \equiv \operatorname{inv} \hat{p}^{\sigma}\left(A \otimes_{K_{1}} N_{1} N_{2}\right)(\bmod 1)$ where $\hat{P}$ is an $N_{1} N_{2}$-prime. Therefore $\left|\left(N_{1} N_{2}\right) \hat{p}:\left(N_{2}\right) p\right| i n v_{P}\left(A \otimes_{K_{1}} N_{2}\right)$ $\equiv \mathrm{b}\left|\left(\mathrm{N}_{1} \mathrm{~N}_{2}\right) \hat{p^{\sigma}:\left(\mathrm{N}_{2}\right)} \mathrm{P}^{\sigma}\right| \operatorname{inv} \mathrm{p}^{\sigma}\left(\mathrm{A} \otimes_{\mathrm{K}_{1}} \mathrm{~N}_{2}\right)(\bmod 1)$. But since $\mathrm{N}_{1} \mathrm{~N}_{2} / \mathrm{N}_{2}$ and $\mathrm{N}_{2} / \mathrm{F}$ are normal and g.c.d. $\left(\mathrm{m},\left|\mathrm{N}_{1} \mathrm{~N}_{2}: \mathrm{N}_{2}\right|\right)=1$ then $\operatorname{inv}_{\mathrm{p}}\left(\mathrm{A} \otimes_{\mathrm{K}_{1}} \mathrm{~N}_{2}\right) \equiv \mathrm{b} \operatorname{inv} \sigma\left(\mathrm{A} \otimes_{\mathrm{K}_{1}} \mathrm{~N}_{2}\right)(\bmod 1)$. This means $\left[A \theta_{K_{l}} N_{2}\right] \in U_{F}\left(N_{2}\right)$; i.e. $\left[A \otimes_{K_{1}} K_{1} K_{2}\right] \in U_{F}\left(K_{1} K_{2}\right)$ Q.E.D.

4. EMBEDDINGS IN SIMPLE ALGEBRAS AND EXTENSIONS OF AUTOMORPHISMS.

Let $\mathrm{K} / \mathrm{F}$ be an extension of number fields, and let $\mathrm{n}$ be a fixed positive integer. If $D$ is a division algebra with [D] $\epsilon B(K)$ then we say that $D$ is (n,F)-adequate if there exists an F-division algebra $B$ with $F \cdot I \subseteq D \subseteq M_{n}(B)$ where $I$ is the identity matrix of $M_{n}(B)$, the full ring of $n \times n$ matrices with entries from B. This concept generalizes that of Fein et al [18] which is the $n=1$ case. We now proceed to obtain results linking $U_{F}(K)$ and $(n, F)$-adequacy via the arithmetic of $F$ and $K$. The $f i r s t$ 
result is a generalization of Fein [22, Proposition 3, p.438].

LEMMA 4.1. Let $\mathrm{K} / \mathrm{F}$ be an extension of number fields with $\mathrm{L}$ being the normal closure of $\mathrm{K} / \mathrm{F}$. Suppose [D] $\in B(K)$ where $D$ is a division ring of index $m$ such that g.c.d. $(m,|L: K|)=1$. If $D$ is $(n, F)$-adequate for a given positive integer $n$ then $\operatorname{inv}_{\hat{p}}\left(D Q_{K} L\right)=\operatorname{inv}_{\hat{O}}\left(\mathbb{D} Q_{K} L\right)$ for all L-primes $\hat{P}$ and $\hat{q}$ such that $\hat{p} \cap F=\hat{Q} \cap F$.

PROOF. Let $A=M_{n}(B)$ where $D$ is embedded in $A$. If $C=C_{A}(K)$ denotes the centralizer of $K$ in $A$ then by Reiner $\left[17\right.$, Corollary 7.14, p.96] we have $[C]=\left[B \otimes_{F} K\right]$. Moreover by Albert [23, Theorem 13, p.53] we have [C] $\left[\theta_{K} \otimes_{1}\right]$ where $D_{1}$ is a division ring with $\left[D_{1}\right] \in B(K)$. Thus $\left[B \otimes_{F} K\right]=\left[D \otimes_{K} D_{l}\right]$ which implies $\left[B \otimes_{F} L\right]=$ $\left[D \otimes_{K} L\right]\left[D_{l} \Theta_{K} L\right]$. Since L/F is: normal then it follows froin Mollin [4, (2.2)-(2.3), p.276] that: $\operatorname{inv}_{\hat{p}}\left(D \otimes_{K} L\right)-\operatorname{inv}_{\hat{Q}}\left(D \otimes_{K} L\right)=\operatorname{inv}_{\hat{Q}}\left(D_{1} \otimes_{K} L\right)-\operatorname{inv}_{\hat{p}}\left(D_{1} \otimes_{K} L\right)$. Since g.c.d. $(|L: K|, m)=1$ then $\operatorname{inv}_{\hat{p}}\left(D \otimes_{K} L\right) \neq \operatorname{inv}_{Q}\left(D \otimes_{K} L\right)$ implies g.c.d. $\left(|D: K|,\left|D_{1}: K\right|\right)$ $\neq 1$ which contradicts that $\mathrm{D} \otimes_{\mathrm{K}} \mathrm{D}_{1}$ is a division algebra, (see (Reiner [17].) We note that the above proof essentially uses the idea of Fein [22].

Now we use Lemma 4.1 to obtain a generalization of Mollin [15, Theorem 3.2, p.263].

THEOREM 4.2. Suppose $K_{i} / F$ for $i=1,2$ are extensions of number fields with $N_{1}$ being the normal closure of $K_{1} / F$ and assume that $K_{1} K_{2} / F$ is normal. Suppose $D$ is a division algebra with [D] $\in U_{F}\left(K_{1}\right)$, and which has index $m$. Suppose $D$ is $\left(n, K_{2}\right)-$ adequate for a given positive integer $n$ such that g.c.d. $\left(m,\left|N_{1} K_{2}: K_{1} K_{2}\right|\right)=1$. If $g=$ g.c.d. $\left(\left|\mathrm{N}_{1}: \mathrm{K}_{1}\right|, \mathrm{m}\right)$ then $\epsilon_{\mathrm{m} / \mathrm{g}}$ is in $\mathrm{K}_{2}$.

PROOF. By Theorem 3.8 we have that $\left[D \Theta_{K_{1}} K_{1} K_{2}\right] \in U_{F}\left(K_{1} K_{2}\right)$, and so we have $U_{F}\left(K_{1} K_{2}\right) \subseteq U_{K_{2}}\left(K_{1} K_{2}\right)$. Now let $P$ and $Q$ be any two $K_{1} K_{2}$-primes with $P \cap K_{2}=2 \cap K_{2}$. Now we invoke Lemma 4.1 to get that $\operatorname{inv}_{P}\left(D \otimes_{K_{1}} K_{1} K_{2}\right)=\operatorname{inv}_{2}\left(D \otimes_{K_{1}} K_{1} K_{2}\right)$. Therefore since $\mathrm{K}_{1} \mathrm{~K}_{2} / \mathrm{F}$ is normal then we may invoke Corollary 3.4 to get $P \cap \mathrm{K}_{2}\left(\epsilon_{\mathrm{m} / \mathrm{g}}\right)=$ $2 \cap \mathrm{K}_{2}\left(\epsilon_{\mathrm{m} / \mathrm{g}}\right)$. However $P$ and 2 were arbitrarily chosen subject only to $P \cap \mathrm{K}_{2}=2 \cap \mathrm{K}_{2}$.

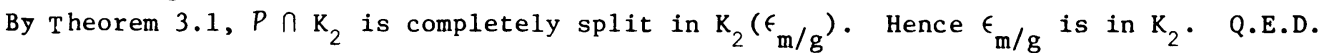

We note that immediate consequences of Theorem 4.2 are Mollin [8, Theorem 3.1, p.175], and Fein et al [24, Theorem 1, p.305]. Moreover the above proof is shorter and more straightforward than the latter two cases.

Finally we present the following generalization of Mollin [10, Theorem 4.5, p.245]. The result is virtually immediate but we present it since it may be of independent interest. Aut(K) (resp. Aut(A)) refers to the automorphism group of K(resp. A).

THEOREM 4.3. Let $K / F$ be an extension of number fields and assume that the fixed field of Aut $(K)$ is contained in $F$. If $[A] \in U_{F}(K)$ with index $n$ then $\sigma \in$ Aut $(K)$ extends to Aut(A) if and only if $\sigma$ fixes $\epsilon_{n}$.

PROOF. Since $U_{F},(K) \subseteq U_{F}(K)$ where $F^{\prime}$ is the fixed field of Aut $(K)$ then the result follows immediately from Mollin [10, Theorem 4.5, p.245]. Q.E.D. We conclude with a note that it is an open question as to whether other results may be generalized to $U_{F}(K)$ when $K / F$ is not normal. Some such examples are Mo11in [6, Theorem 4.5, p.476], [7, Theorem 2.1, p.202], [2, Theorem 1.2, p.262], and [12, Theorem 1, p.1075]. It should also be noted that recently Greenfield [25] has done some work on uniform distribution from a different perspective modelled after Mo11in [15]. 
ACKNOWLEDGEMENT: This research was supported by N.S.E.R.C. Canada.

\section{REFERENCES}

1. M. BENARD and M. SCHACHER, The Schur Subgroup II, J. Algebra 22 (1972), 378-385.

2. R.A. MOLLIN, Uniform Distribution and the Schur Subgroup, J.Algebra, 42, No. 1, (1976), 261-277.

3. R.A. MOLLIN, Uniform Distribution and Real Fields, J. Algebra 43, No. 1, (1976), 155-167.

4. R.A. MOLLIN, Algebras with Uniformly Distributed Invariants, J. Algebra 44, No. 1, (1977), 271-282.

5. R.A. MOLLIN, $U(\mathrm{~K})$ For Quadratic Fields K, Communications in Algebra 4 (8), (1976), 747-759.

6. R.A. MOLLIN, The Schur Group of a Field of rharacteristic Zero, Pacific J. Math. 76 $(2),(1978), 471-478$.

7. R.A. MOLLIN, Uniform Distribution Classified, Math. Z. 165, (1979), 199-211.

8. R.A. MOLLIN, Induced p-Elements in the Schur Group, Pacific J. Math. 90. No. 1 (1980), 169-176.

9. R.A. MOLLIN, Induced Quaternion Algebras in the Schur Group, Can. J. Math. $\underline{33}$ (6), (1981), 1370-1379.

10. R.A. MOLLIN, Herstein's Conjecture, Automorphisms and the Schur Group. Communjcations in Algebra 6 (3), (1978), 237-248.

11. R.A. MOLLIN, Splitting Fields and Group Characters, J. reine angew Math. $\underline{315}$ (1980), 107-114.

12. R.A. MOLliN, Cyclotomic Division Algebras, Can. J. Math. 33 (5), (1981), $1074-1084$.

13. R.A. MOLLIN, Schur Indices, Sums of Squares and Splitting Fields, C.R. Math. Rep. Acad. Sci. Canada, Vol. III (1981) No. 6, 301-306.

14. R.A. MOLLIN, Class Numbers and a Generalized Fermat Theorem, J. Number Theory $\underline{16}$, No. 3, (1983), 420-429.

15. R.A. MOLLIN, Generalized Uniform Distribution of Hasse Invariants, Communications in Algebra 5 (3), (1977), 245-266.

16. M. BENARD, The Schur Subgroup I, J. Algebra 22 (2), (1972), 374-377.

17. I. REINER, Maximal Orders, Academic Press, New York, 1975.

18. B. FEIN and M. SCHACHER, Embedding Finite Groups in Rational Division Algebras, I, J. Algebra 17 (1971), 421-428.

19. D.A. MARCUS, Number Fields, Springer-Verlag, New York, 1977.

20. T. YAMADA, The Schur Subgroup of the Brauer Group, Lecture Notes in Mathematics, No. 397, Springer-Verlag (1974).

21. E. WITT, Die Algebraische Struktur des Gruppenringes einer Endlichen Gruppe uber einem Zahlkorper, J. Reine angew Math. 190 (1952), 231-245.

22. B. FEIN, Embedding Rational Division Algebras, Proc. Amer. Math. Soc. 32, (2), (1972), 427-429. 
23. A.A. ALBERT, Structure of Algebras, Amer. Math. Soc., Providence, R.I. 1961.

24. B. FEIN and M. SCHACHER, Embedding Finite Groups in Rational Division Algebras III, J. Algebra, 28 (1974), 304-310.

25. G. GREENFIELD, Uniform Distribution in Subgroups of the Brauer Group of an Algebraic Number Field, Pacific J. Math. 107 (2) (1983), 369-381. 


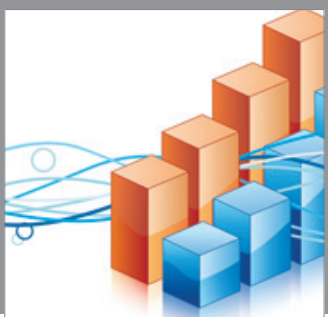

Advances in

Operations Research

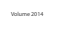

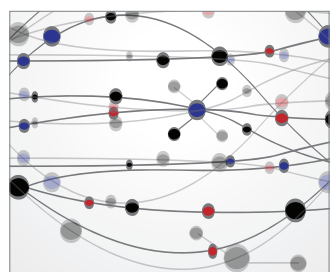

\section{The Scientific} World Journal
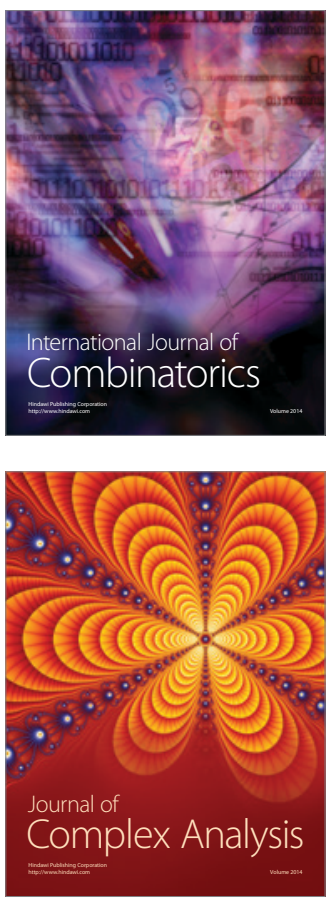

International Journal of

Mathematics and

Mathematical

Sciences
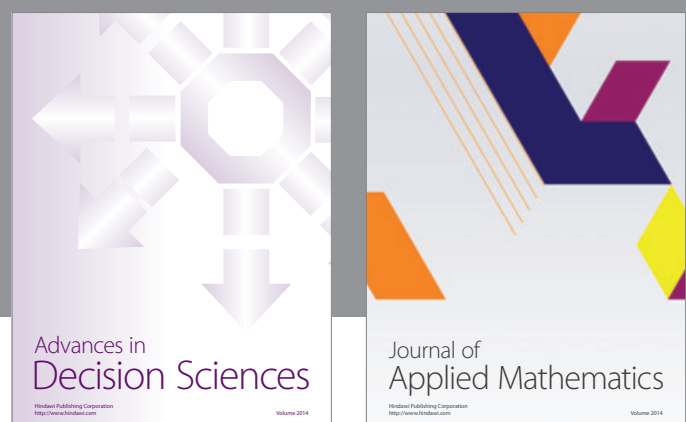

Journal of

Applied Mathematics
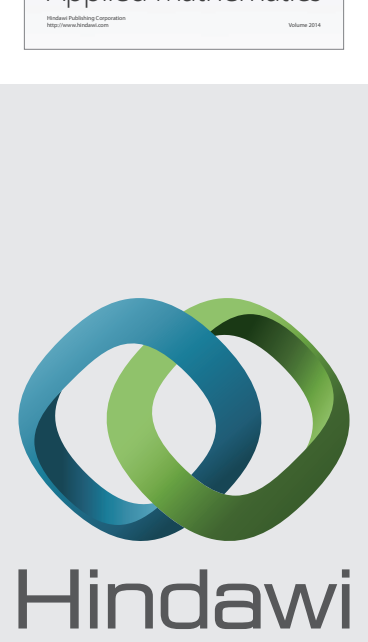

Submit your manuscripts at http://www.hindawi.com
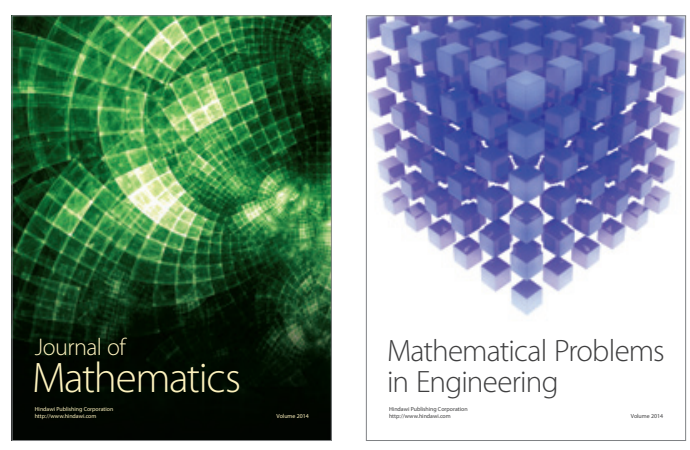

Mathematical Problems in Engineering
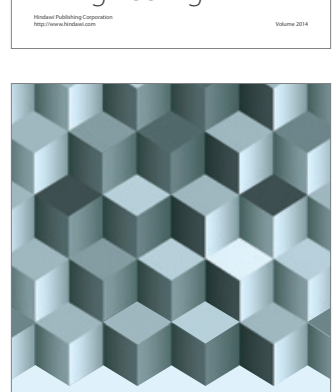

Journal of

Function Spaces
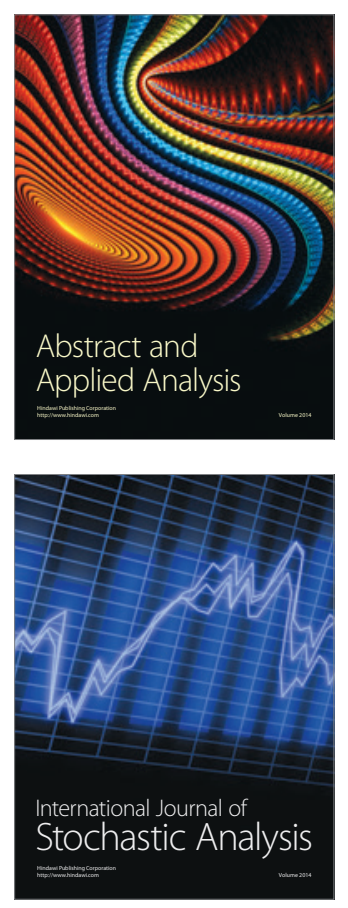

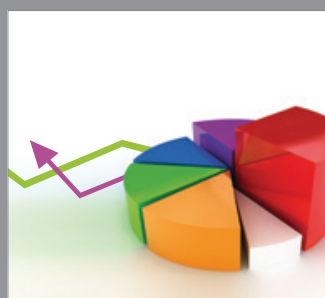

ournal of

Probability and Statistics

Promensencen
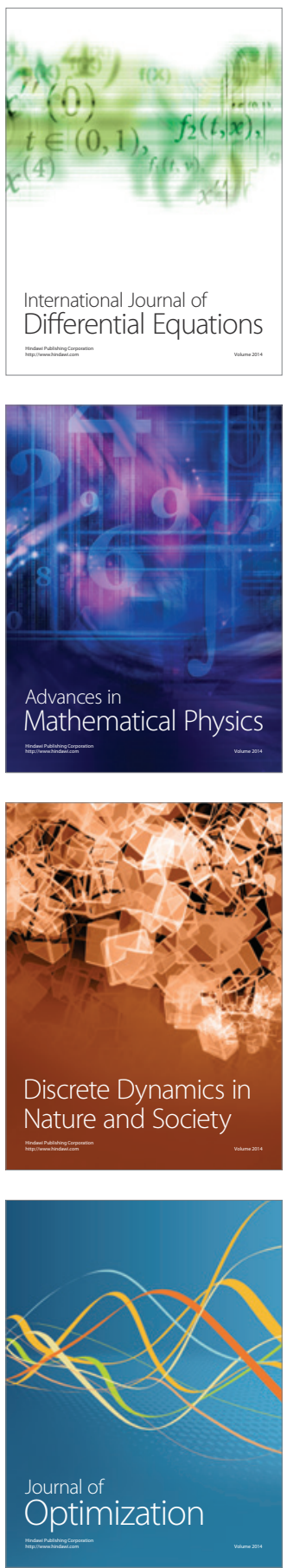